\title{
Symmetry Reduced and New Exact Nontraveling Wave Solutions of $(2+1)$-Dimensional Potential Boiti-Leon-Manna-Pempinelli Equation
}

\author{
Chen Han-Lin and Xian Da-Quan \\ School of Sciences, Southwest University of Science and Technology, Mianyang, Sichuan 621010, China \\ Correspondence should be addressed to Xian Da-Quan; daquanxian@163.com
}

Received 21 September 2012; Accepted 8 December 2012

Academic Editor: Zhengde Dai

Copyright (c) 2013 C. Han-Lin and X. Da-Quan. This is an open access article distributed under the Creative Commons Attribution License, which permits unrestricted use, distribution, and reproduction in any medium, provided the original work is properly cited.

With the aid of Maple symbolic computation and Lie group method, (2+1)-dimensional PBLMP equation is reduced to some (1+1)dimensional PDE with constant coefficients. Using the homoclinic test technique and auxiliary equation methods, we obtain new exact nontraveling solution with arbitrary functions for the PBLMP equation.

\section{Introduction}

In this paper, we will consider the potential Boiti-LeonManna-Pempinelli (PBLMP) equation

$$
u_{y t}+u_{x x x y}-3 u_{x x} u_{y}-3 u_{x} u_{x y}=0
$$

where $u: R_{x} \times R_{y} \times R_{t}^{+} \rightarrow R$. By some transformations, the PBLMP equation (1) can be equivalent to the asymmetric Nizhnik-Novikov-Veselov (ANNV) system. In fact, the ANNV equation can be obtained from the inner parameterdependent symmetry constraint of the KP equation [1] and may be considered as a model for an incompressible fluid [2]. The Painlevé analysis, Lax pair, and some exact solutions have been studied for the PBLMP equation [3]. Tang and Lou obtained the bilinear form of (1) and variable separation solutions including two arbitrary functions by the multilinear variable separation approach $[4,5]$.

In this paper, by means of Maple symbolic computation, we will use the Lie group method $[6,7]$, homoclinic test technique $[8,9]$ and so forth to reduce and solve the PBLMP equation. First, we will derive symmetry of (1). Then we use the symmetry to reduce $(1)$ to some $(1+1)$-dimensional PDE with constant coefficients. Finally, solving the reduced PDE by Homoclinic test technique and auxiliary equation methods $[10,11]$ implies abundant exact nontraveling wave periodic solutions for the PBLMP equation.

\section{Symmetry of (1)}

This section is devoted to Lie point group symmetries of (1). Let

$$
\sigma=\sigma\left(x, y, t, u, u_{x}, u_{y}, u_{t}\right)
$$

be the symmetry of (1). Based on Lie group theory [6], $\sigma$ satisfies the following symmetry equation:

$$
\sigma_{y t}+\sigma_{y x x x}-3 u_{x x} \sigma_{y}-3 u_{y} \sigma_{x x}-3 u_{x} \sigma_{x y}-3 u_{x y} \sigma_{x}=0 .
$$

To get some symmetries of (1), we take the function $\sigma$ in the form

$$
\begin{aligned}
\sigma= & a(x, y, t) u_{x}+b(x, y, t) u_{y}+c(x, y, t) u_{t} \\
& +d(x, y, t) u+e(x, y, t),
\end{aligned}
$$

where $a, b, c, d, e$ are functions of $x, y, t$ to be determined, and $u(x, y, t)$ satisfies (1). Substituting (4) and (1) into (3), one can get

$$
\begin{gathered}
a=\frac{1}{3} k_{1} x+\lambda(t), \quad b=\mu(y), \\
c=k_{1} t+k_{2}, \quad d=\frac{1}{3} k_{1}, \quad e=\frac{1}{3} \lambda^{\prime}(t) x+\xi(t),
\end{gathered}
$$


where $k_{1}, k_{2}$ are arbitrary constants. $\lambda(t), \xi(t)$ are arbitrary functions of $t . \mu(y)$ is a arbitrary function of $y$. Substituting (5) into (4), we obtain the symmetries of (1) as follows:

$$
\begin{aligned}
\sigma= & \left(\frac{1}{3} k_{1} x+\lambda(t)\right) u_{x}+\mu(y) u_{y}+\left(k_{1} t+k_{2}\right) u_{t}+\frac{1}{3} k_{1} u \\
& +\frac{1}{3} \lambda^{\prime}(t) x+\xi(t) .
\end{aligned}
$$

\section{Symmetry Reduction of (1)}

Based on the integrability of reduced equation of the symmetry (6), we consider the following three cases.

Case 1. Let $k_{1}=k_{2}=0, \lambda(t)=r, \xi(t)=1, \mu(y)=-1 / \tau(y)$ in (6), then

$$
\sigma=\tau(y)^{-1}\left(r \tau(y) u_{x}-u_{y}+\tau(y)\right),
$$

where $r$ is an arbitrary nonzero constant, $\tau(y) \neq 0$. Solving the differential equation for $\sigma=0$, one gets

$$
u=\int \tau(y) d y+w(\theta, t), \quad \theta=x+\int r \tau(y) d y .
$$

Substituting (8) into (1), we get the following $(1+1)$-dimensional nonlinear PDE with constant coefficients:

$$
r w_{\theta \theta \theta \theta}-6 r w_{\theta} w_{\theta \theta}+r w_{\theta t}-3 w_{\theta \theta}=0
$$

Integrating (9) once with respect to $\theta$ and taking integration constant to zero yield

$$
r w_{\theta \theta \theta}-3 r w_{\theta}^{2}+r w_{t}-3 w_{\theta}=0 .
$$

Case 2. Taking $k_{1}=0, k_{2}=1, \lambda(t)=0, \xi(t)=0, \mu(y)=$ $1 / \tau(y)$ in (6) yields

$$
\sigma=\tau(y)^{-1}\left(u_{y}+r \tau(y) u_{t}\right) .
$$

Solving the differential equation for $\sigma=0$, one gets

$$
u=w(x, \theta), \quad \theta=t-\int \tau(y) d y
$$

Substituting (12) into (1), we have the function $w(x, \theta)$ which must satisfy the following PDE:

$$
w_{x x x \theta}-3 w_{x x} w_{\theta}-3 w_{x} w_{x \theta}+w_{\theta \theta}=0 .
$$

Case 3. Let $k_{1}=k_{2}=0, \lambda(t)=1, \xi(t)=0, \mu(y)=-1 / \tau(y)$ in (6), then

$$
\sigma=\tau(y)^{-1}\left(\tau(y) u_{x}-u_{y}\right)
$$

Solving the equation for $\sigma=0$, we obtain

$$
u=w(\theta, t), \quad \theta=x+\int \tau(y) d y .
$$

Substituting (15) into (1) yields a reduced PDE of (1) with constant coefficients:

$$
w_{\theta \theta \theta \theta}-6 w_{\theta \theta} w_{\theta}+w_{\theta t}=0
$$

Integrating (16) once with respect to $\theta$ and taking integration constant to zero yield

$$
w_{\theta \theta \theta}-3 w_{\theta}^{2}+w_{t}=0
$$

Combining the above results, we obtain some reduced equations of (1) expressed by (10), (13), and (17), respectively. Meanwhile many new explicit solutions of (1) from these reduced Equations. can be achieved. We omit other cases based on symmetries (6) here.

\section{Solve Reduced PDE and Get Exact Nontraveling Wave Solutions of (1)}

In this section, we seek exact nontraveling wave solutions of (1) by using some appropriate methods to solve reduced equations (10), (13), and (17).

4.1. Solve Reduced PDE (10). Now, we seek solutions of (10) by auxiliary equation method. Make transformation as follows:

$$
w(\theta, t)=\varphi(\xi), \quad \xi=p \theta+q t,
$$

where $p, q$ are nonzero constants. Substituting (18) into (10) obtains an ordinary differential equation for $\varphi(\xi)$ as follows:

$$
p^{3} r \varphi^{\prime \prime \prime}-3 r p^{2} \varphi^{\prime 2}+(q r-3 p) \varphi^{\prime}=0,
$$

where $\varphi^{\prime}=d \varphi / d \xi$. Let $\varphi^{\prime}=f$, then (19) can be written as

$$
p^{3} r f^{\prime \prime}-3 r p^{2} f^{2}+(q r-3 p) f=0
$$

This is the fourth type of ellipse equation (12), its solutions are as follows:

$$
f=\left\{\begin{array}{l}
-\frac{3 p-q r}{2 p^{2} r} \operatorname{sech}^{2}\left[\sqrt{\frac{3 p-q r}{4 p^{3} r}}\left(\xi-\xi_{0}\right)\right], \\
\frac{3 p-q r}{2 p^{2} r} \operatorname{csch}^{2}\left[\sqrt{\frac{3 p-q r}{4 p^{3} r}}\left(\xi-\xi_{0}\right)\right], \\
p r(3 p-q r)>0, \\
\left.-\frac{3 p-q r}{2 p^{2} r} \sec ^{2}\left[\sqrt{\frac{-\frac{3 p-q r}{4 p^{3} r}}{p r}(3 p-q r)}, \xi_{0}\right)\right],
\end{array}\right.
$$


where $\xi_{0}$ is the integration constant. From the result of (21), some new exact solutions $u_{1}$ through $u_{3}$ of (1) can be obtained:

$$
\begin{aligned}
& u_{1}=\int \tau(y) d y-\sqrt{\frac{3 p-q r}{p r}} \\
& \times \tanh \left[\sqrt{\frac{3 p-q r}{4 p^{3} r}}\left(p\left(x+r \int \tau(y) d y\right)+q t-\xi_{0}\right)\right], \\
& \operatorname{pr}(3 p-q r)>0 \text {, } \\
& u_{2}=\int \tau(y) d y-\sqrt{\frac{3 p-q r}{p r}} \\
& \times \operatorname{coth}\left[\sqrt{\frac{3 p-q r}{4 p^{3} r}}\left(p\left(x+r \int \tau(y) d y\right)+q t-\xi_{0}\right)\right] \text {, } \\
& p r(3 p-q r)>0 \text {, } \\
& u_{3}=\int \tau(y) d y-\sqrt{\frac{q r-3 p}{p r}} \\
& \times \tan \left[\sqrt{\frac{q r-3 p}{4 p^{3} r}}\left(p\left(x+r \int \tau(y) d y\right)+q t-\xi_{0}\right)\right], \\
& \operatorname{pr}(q r-3 p)<0 \text {. }
\end{aligned}
$$

Particularly, we assume $p=q=1, r=2, \tau(y)=\sin (y)$, $\xi_{0}=0, x=\operatorname{sech}(t)$, then the solution $u_{1}$ can be depicted by Figure 1(a). If $p=-1, q=1, r=-1, \tau(y)=\mp \cos (y), \xi_{0}=$ $0, x=\sin (\mathrm{t})$, then $u_{3}$ can be depicted by Figures $1(\mathrm{~b})$ and $2(\mathrm{a})$.

4.2. Solve Reduced PDE (13). Make transformation to (13) as follows:

$$
w(x, \theta)=\varphi(\xi), \quad \xi=k x+c \theta
$$

where $k, c$ are non-zero constants. Substituting (23) into (13) then we have

$$
c \varphi^{\prime}+k^{3} \varphi^{\prime \prime \prime}-3 K^{2} \varphi^{\prime 2}=0
$$

It is equivalent to (19). Based on the above accordant idea, we can get

$$
\begin{aligned}
u_{4}= & \sqrt{-\frac{c}{2 k}} \\
& \times \tanh \left[\sqrt{-\frac{c}{2 k^{3}}}\left(k x+c\left(t-\int \tau(y) d y\right)-\xi_{0}\right)\right], \quad k c<0, \\
u_{5}= & \sqrt{-\frac{c}{2 k}} \\
& \times \operatorname{coth}\left[\sqrt{-\frac{c}{2 k^{3}}}\left(k x+c\left(t-\int \tau(y) d y\right)-\xi_{0}\right)\right], \quad k c<0,
\end{aligned}
$$

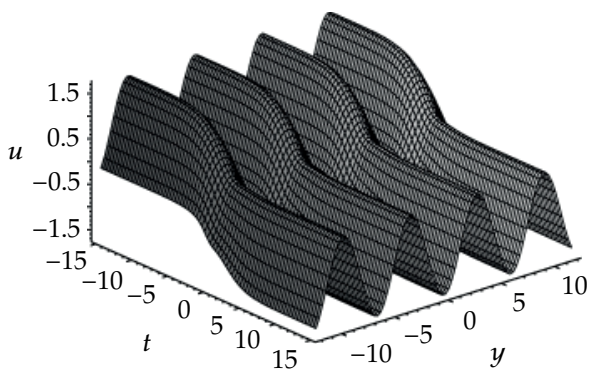

(a)

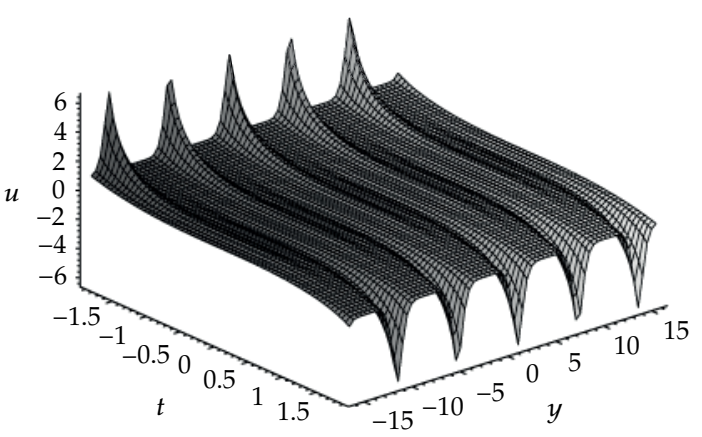

(b)

Figure 1: (a) The figure of $u_{1}$ as $p=1, q=1, r=2, \tau(y)=$ $\sin (y), \xi_{0}=0, x=\operatorname{sech}(t)$. (b) The figure of $u_{3}$ as $p=-1, q=$ $1, r=-1, \tau(y)=-\cos (y), \xi_{0}=0, x=\sin (t)$.

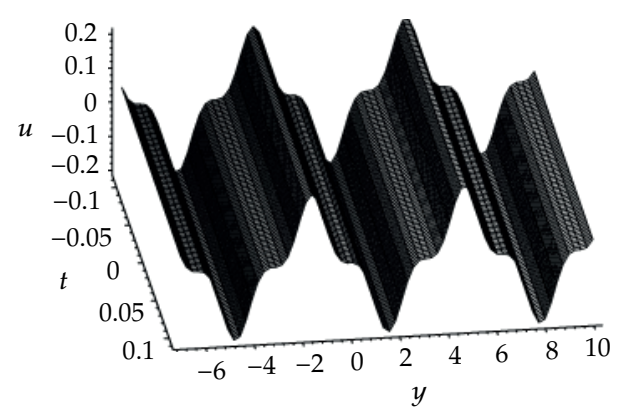

(a)

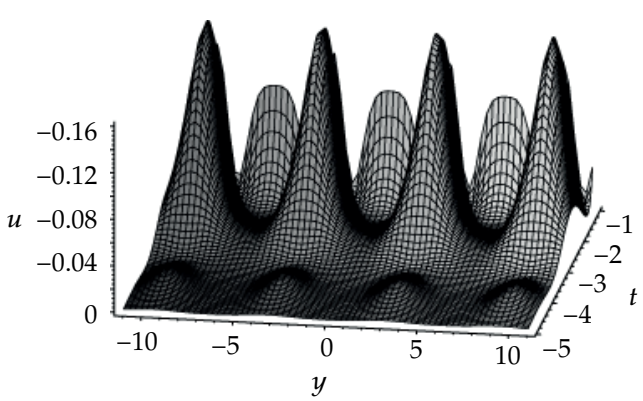

(b)

Figure 2: (a) The figure of $u_{3}$ as $p=-1, q=1, r=-1, \tau(y)=$ $\cos (y), \xi_{0}=0, x=\sin (t)$. (b) The figure of $u_{9}$ as $p_{1}=1, c_{1}=$ $1, p_{2}=1, \tau(y)=\sin (y), x=\sin (t)$. 


$$
\begin{aligned}
u_{6}= & \sqrt{\frac{c}{2 k}} \\
& \times \tan \left[\sqrt{\frac{c}{2 k^{3}}}\left(k x+c\left(t-\int \tau(y) d y\right)-\xi_{0}\right)\right], \quad k c>0 .
\end{aligned}
$$

4.3. Solve Reduced PDE (17). In this section, we use homoclinic test technique $[8,9]$ to (17) and transform the unknown function as follows:

$$
w(\theta, t)=-2(\ln f(\theta, t))_{\theta} .
$$

Substituting (26) into (17) and using the bilinear form, we can get

$$
\left(D_{\theta} D_{t}+D_{\theta}^{4}\right)(f \cdot f)=0
$$

where the Hirota operator $D$ is defined in [12]. In this case we choose extended homoclinic test function

$$
f=e^{-p_{1}\left(\theta-\omega_{1} t\right)}+c_{1} \cos \left(p_{2}\left(\theta+\omega_{2} t\right)\right)+c_{2} e^{p_{1}\left(\theta-\omega_{1} t\right)},
$$

where $p_{2}, \omega_{1}, \omega_{2}, c_{1}$, and $c_{2}$ are real constants to be determined. Substituting (28) into (27) yields a set of algebraic equations as follows:

$$
\begin{gathered}
p_{1} c_{1} p_{2}\left(4\left(p_{1}^{2}-p_{2}^{2}\right)+\omega_{2}-\omega_{1}\right)=0, \\
c_{1}\left(\left(p_{1}^{4}+p_{4}^{4}-6 p_{1}^{2} p_{2}^{2}\right)-p_{1}^{2} \omega_{1}-p_{2}^{2} \omega_{2}\right)=0, \\
p_{1} p_{2} c_{1} c_{2}\left(4\left(p_{1}^{2}-p_{2}^{2}\right)+\omega_{2}-\omega_{1}\right)=0, \\
c_{1} c_{2}\left(\left(p_{1}^{4}+p_{4}^{4}-6 p_{1}^{2} p_{2}^{2}\right)-p_{1}^{2} \omega_{1}-p_{2}^{2} \omega_{2}\right)=0, \\
4\left(4 p_{1}^{4} c_{2}+c_{1}^{2} p_{2}^{4}\right)-4 p_{1}^{2} \omega_{1} c_{2}-c_{1}^{2} p_{2}^{2} \omega_{2}=0 .
\end{gathered}
$$

Solving the above equations (29) yields

(1) $\left\{\begin{array}{l}p_{1}=p_{1}, \quad p_{2}=p_{2}, \quad c_{1}=0, \quad c_{2}=c_{2}, \\ \omega_{1}=4 p_{1}^{2}, \quad \omega_{2}=\omega_{2},\end{array}\right.$

(2) $\left\{\begin{array}{l}p_{1}=p_{1}, \quad p_{2}=p_{2}, \quad c_{1}=c_{1}, \quad c_{2}=-\frac{c_{1}^{2} p_{2}^{2}}{p_{1}^{2}}, \\ \omega_{1}=-3 p_{2}^{2}+p_{1}^{2}, \quad \omega_{2}=-3 p_{1}^{2}+p_{2}^{2},\end{array}\right.$

(3) $\left\{\begin{array}{l}p_{1}=p_{2} i, \quad p_{2}=p_{2}, \quad c_{1}=c_{1}, \quad c_{2}=c_{2}, \\ \omega_{1}=-4 p_{2}^{2}, \quad \omega_{2}=4 p_{2}^{2},\end{array}\right.$

(4) $\left\{\begin{array}{l}p_{1}=p_{2} i, \quad p_{2}=p_{2}, \quad c_{1}=c_{1}, \quad c_{2}=\frac{1}{4} c_{1}^{2}, \\ \omega_{1}=\omega_{2}-8 p_{2}^{2}, \quad \omega_{2}=\omega_{2},\end{array}\right.$ where $i^{2}=-1$. Substituting (30)-(33) into (28) yields the solutions $u_{7}$ through $u_{11}$ of (1) as follows:

$$
u_{7}=-2 p_{1} \tanh \left(p_{1}\left(x+\int \tau(y) d y\right)-4 p_{1}^{2} t+\frac{1}{2} \ln c_{2}\right),
$$

when $c_{2}>0$ in (30);

$$
u_{8}=-2 p_{1} \operatorname{coth}\left(p_{1}\left(x+\int \tau(y) d y\right)-4 p_{1}^{2} t+\frac{1}{2} \ln \left(-c_{2}\right)\right),
$$

when $c_{2}<0$ in $(30)$;

$$
\begin{aligned}
& u_{9}=-2 p_{1} p_{2} \\
& \times\left(\operatorname { c o t h } \left(p_{1}\left(x+\int \tau(y) d y\right)\right.\right. \\
& \left.\quad-\left(p_{1}^{2}-3 p_{2}^{2}\right) t+\ln \frac{c_{1} p_{2}}{p_{1}}\right) \\
& \left.\quad+\sin \left(p_{2}\left(x+\int \tau(y) d y\right)-\left(3 p_{1}^{2}-p_{2}^{2}\right) t\right)\right) \\
& \times\left(p _ { 2 } \operatorname { s i n h } \left(p_{1}\left(x+\int \tau(y) d y\right)\right.\right. \\
& \left.\quad-\left(p_{1}^{2}-3 p_{2}^{2}\right) t+\ln \frac{c_{1} p_{2}}{p_{1}}\right) \\
& \left.\quad+p_{1} \cos \left(p_{2}\left(x+\int \tau(y) d y\right)-\left(3 p_{1}^{2}-p_{2}^{2}\right) t\right)\right)^{-1}
\end{aligned}
$$

when $c_{1} p_{1} p_{2}>0$ in (31) (see Figure 2(b));

$$
u_{10}(x, y, t)=p_{2} \tan \left(p_{2}\left(x+\int \tau(y) d y\right)+4 p_{2}^{2} t\right),
$$

when $c_{2}=1$ in (32);

$$
\begin{aligned}
& u_{11}(x, y, t) \\
& =-2 p_{2} \\
& \times \frac{\sin \left(p_{2}\left(x+\int \tau(y) d y\right)+\left(8 p_{2}^{2}-\omega_{2}\right) t\right)+\sin \left(p_{2}\left(x+\int \tau(y) d y\right)+\omega_{2} t\right)}{\cos \left(p_{2}\left(x+\int \tau(y) d y\right)+\left(8 p_{2}^{2}-\omega_{2}\right) t\right)+\cos \left(p_{2}\left(x+\int \tau(y) d y\right)+\omega_{2} t\right)}, \\
& \text { when } c_{1}=2 \text { in (33). }
\end{aligned}
$$

Remark 1. If one lets $w_{\theta}=v$ in (16), then (16) can be written as

$$
v_{t}-6 v v_{\theta}+v_{\theta \theta \theta}=0 .
$$

This is the famous KdV equation.

\section{Conclusions}

In this paper, a combination of Lie group method and homoclinic test technique and so forth is applied and thus the 
symmetries (6) are obtained. The (2+1)-dimensional potential Boiti-Leon-Manna-Pempinelli equation (1) is reduced to $(1+1)$-dimensional nonlinear PDE of constant coefficients (10), (13), and (17). Further auxiliary equation method and homoclinic test technique are used and some new exact nontraveling wave solutions are obtained. And they include some special and strange structures to be further studied and other relevant solutions about symmetry (6) will be discussed later in another paper. Our results show that combining the Lie group method with homoclinic test technique and so forth is effective in finding nontraveling wave exact solutions of nonlinear evolution equations.

\section{Acknowledgments}

The authors would like to thank Professor Dai Zhengde for his helpful discussions. This work was supported by the Chinese Natural Science Foundation Grant no. 10971169 and the key research projects of Sichuan Provincial Educational Administration, no. 10ZA021.

\section{References}

[1] S.-Y. Lou and X.-B. Hu, "Infinitely many Lax pairs and symmetry constraints of the KP equation," Journal of Mathematical Physics, vol. 38, no. 12, pp. 6401-6427, 1997.

[2] M. J. Ablowitz and P. A. Clarkson, Solitons, Nonlinear Evolution Equations and Inverse Scattering, vol. 149, Cambridge University Press, Cambridge, Mass, USA, 1991.

[3] P. G. Estévez and S. Leble, "A wave equation in $2+1$ Painlevé analysis and solutions," Inverse Problems, vol. 11, no. 4, pp. 925937, 1995.

[4] X. Y. Tang, Locallized Excitations and Symmetries of $(2+1)$ Dimensional Nonlinear Systems, Shanghai Physics Department, Shanghai Jiao Tong University, Shanghai, China, 2004.

[5] S. Y. Lou and X. Y. Tang, Methods of Nonlinear Mathematical Physics, Beling Science Press of China, Beijing, China, 2006.

[6] P. J. Olver, Applications of Lie Groups to Differential Equations, vol. 107, Springer, New York, NY, USA, 1986.

[7] D. Q. Xian, "New exact solutions to a class of nonlinear wave equations," Journal of University of Electronic Science and Technology of China, vol. 35, no. 6, pp. 977-980, 2006.

[8] Z. Dai and D. Xian, "Homoclinic breather-wave solutions for Sine-Gordon equation," Communications in Nonlinear Science and Numerical Simulation, vol. 14, no. 8, pp. 3292-3295, 2009.

[9] Z.-D. Dai, D.-Q. Xian, and D.-L. Li, "Homoclinic breather-wave with convective effect for the (1+1)-dimensional boussinesq equation," Chinese Physics Letters, vol. 26, no. 4, Article ID 040203, 2009.

[10] H. L. Chen and D. Q. Xian, "Periodic wave solutions for the Klein-Gordon-Zakharov equation," Acta Mathematicae Applicatae Sinica, vol. 29, no. 6, 2006.

[11] S. K. Liu and S. D. Liu, Nonliner Equations in Physics, Peking University Press, Beijing, China, 2000.

[12] R. Hirota, "Exact envelope-soliton solutions of a nonlinear wave equation," Journal of Mathematical Physics, vol. 14, no. 7, pp. 805-809, 1973. 


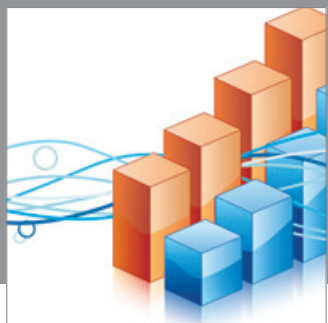

Advances in

Operations Research

mansans

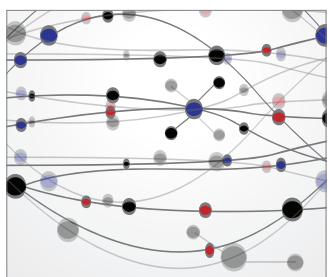

The Scientific World Journal
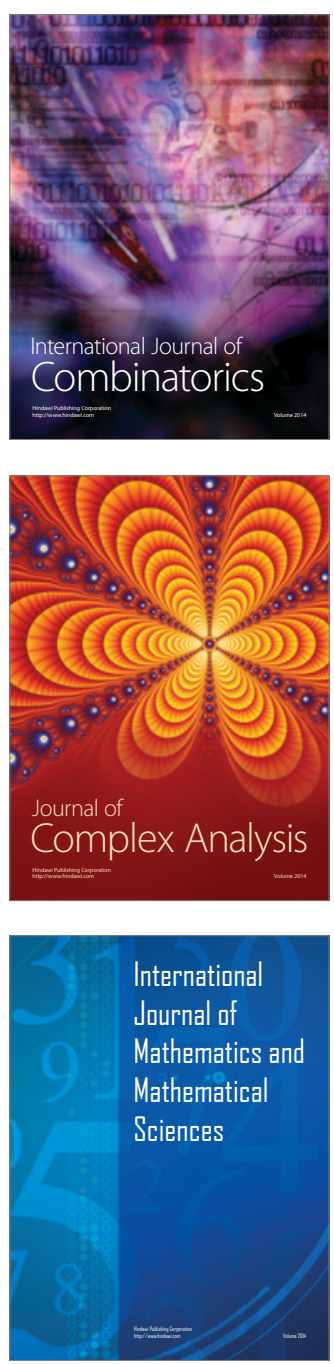
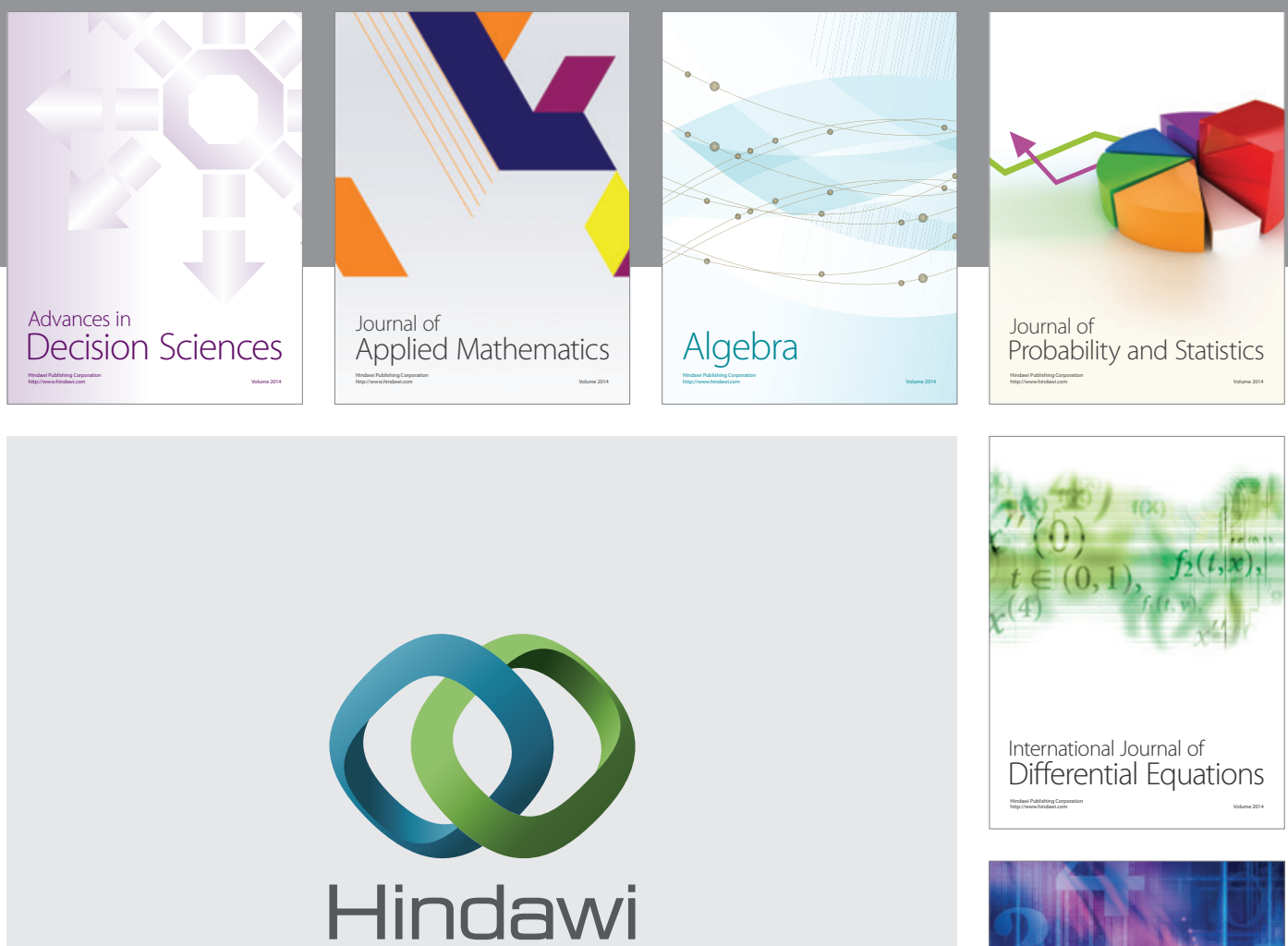

Submit your manuscripts at http://www.hindawi.com
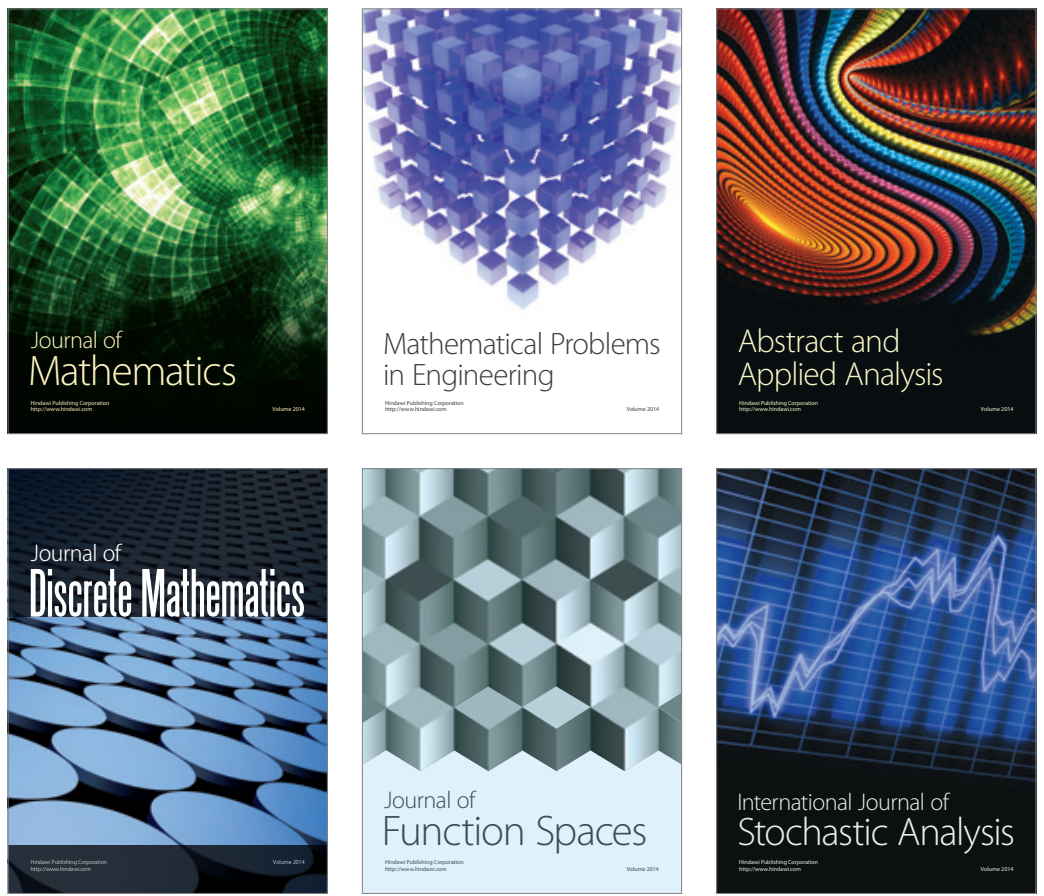

Journal of

Function Spaces

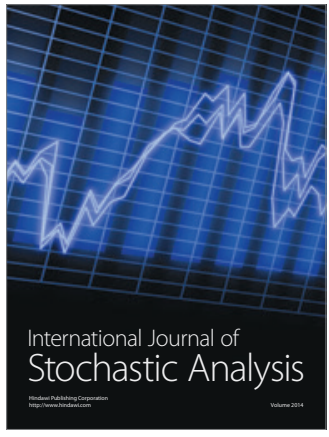

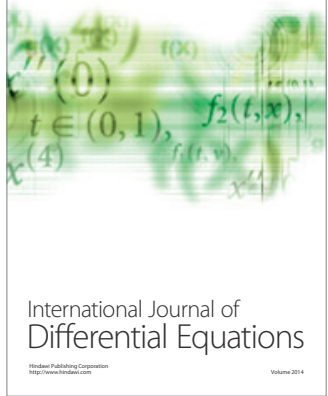
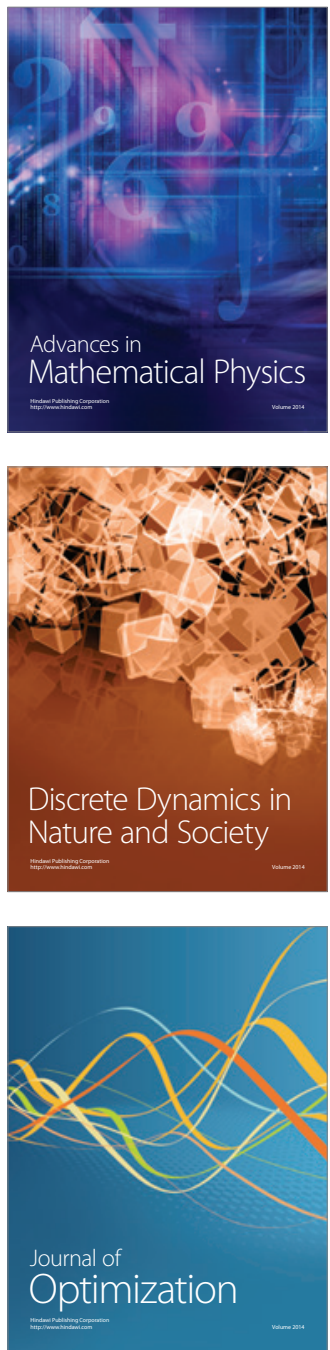